\title{
EDQM-HC Transformation Terminology
}

National Cancer Institute

\section{Source}

National Cancer Institute. EDQM-HC Transformation Terminology. NCI Thesaurus. Code C150117.

Terminology subset about procedures that are carried out in order to convert a

manufactured item that requires such a procedure into a pharmaceutical product, i.e., from its manufactured dose form to its administrable dose form. Note: A transformation is not required when the manufactured item is equal to the pharmaceutical product. 